\title{
LOS PROBLEMAS DE CONVIVENCIA ESCOLAR: PERCEPCIONES, FACTORES Y ABORDAJES EN EL AULA
}

\author{
Martha Liliana Palomino Leiva, ${ }^{42}$ \\ Amparo Dagua Paz ${ }^{43}$
}

\begin{abstract}
RESUMEN
La presente investigación fue realizada en la institución educativa Limbania Velasco, municipio Santander de Quilichao, (Cauca- Colombia), con estudiantes y directores de grupo de los grados terceros, jornada mañana y grados octavos, jornada tarde en el periodo lectivo 2008 - 2009. El objetivo general se centró en analizar los factores que limitan la convivencia escolar con el fin de validar prácticas y generar estrategias psicosociales que promuevan relaciones interpersonales adecuadas hacia una cultura de paz al interior de las aulas escolares. La metodología utilizada fue cualitativa fundamentada en la investigación acción, con una muestra de 109 estudiantes (79 de los grados terceros y 80 de los grados octavos). Entre las estrategias de investigación se destaca la visita institucional, la observación de aula de clase, de recreo y de juego, la entrevista individual y grupal, el taller, la revisión de los observadores de aula y los encuentros de integración titulados convivencias escolares.
\end{abstract}

Como resultados principales se encuentra que docentes y estudiantes perciben la convivencia en la institución como positiva aunque se presenten algunos problemas de relación y de indisciplina al interior de las aulas escolares. En los grados terceros se identifica la necesidad de fortalecer los espacios de diálogo, escucha y participación; los estudiantes de los grados octavos relacionan la convivencia con sus vivencias y experiencias cotidianas, algunos la resaltan positiva y otros negativamente; los docentes manifiestan la importancia de abordar la actitud del estudiante y sus emociones. Se concluye que los problemas de convivencia escolar son diversos, complejos y dinámicos. Estos tienen una connotación cultural y normativa. Al ser abordados a partir de lo psicosocial implican mirar las interacciones desde el contexto familiar y social. La comunidad educativa desarrolla estrategias y acciones positivas y negativas que requieren ser reflexionadas, se definen estrategias para intervenir problemas de actitud personal y grupal, comunicación interpersonal, la tramitación adecuada del conflicto y el trabajo cooperativo.

Palabras clave: convivencia escolar, interacciones, comunidad educativa, normatividad, intervención psicosocial.

42 Especialista en Gerencia Educativa con énfasis en Gestión de proyectos. Investigadora principal del Grupo de Investigacion Social GIS. Tutora Unad y líder local Escuela Ciencias de la Educación en el ceres Santander de Quilichao, Cauca.

43 Especialista en Gerencia Educativa con énfasis en Gestión de proyectos. Co investigadora del Grupo de Investigación Social GIS. Tutora Unad y líder local Escuela Ciencias Sociales, artes y humanidades en el Ceres Santander de Quilichao, Cauca. 


\begin{abstract}
This research was carried out in the Limbania Velasco Educational Institution, located in Santander de Quilichao, Cauca, Colombia, with students and group leaders of third grade, morning session, and eighth grade, afternoon session, during the 2008-2009 school year. The general goal was to analyze the factors which limit coexistence in schools with the purpose of validating psycho-social practices and strategies which promote satisfactory interpersonal relationships leading to a culture of peace in classrooms. A qualitative methodology was employed, based on active investigation on a sample of 159 students (79 from third grade and 80 from eighth grade). The research strategies include an institutional visit, observation of classroom, recess and playing environments, individual and group interviews, workshops, evaluation of classroom observers, and integration sessions or school retreats.
\end{abstract}

The results shows that teachers and students perceive coexistence inside their institution as positive but vulnerable to academic, family, social, interpersonal and disciplinary problems. In third grade classrooms, the need to expand opportunities for dialog, listening and participation among students was identified; in eighth grade classrooms, students relate coexistence with their daily experience; some consider it positive and others, negative; teachers point out the importance of addressing the attitudes and emotions of students. In conclusion, the problems of coexistence in schools are diverse, complex and dynamic. Approaching them from a psycho-social perspective implies examining human interaction in family and social contexts. The educational community develops both positive and negative strategies and actions which must be reflected on. Strategies exist for intervention in problems regarding personal and group attitudes, interpersonal communication, adequate conflict management and collaborative work.

Key words: coexistence in schools, interaction, educational community, regulations, psychosocial intervention.

Recibido: 28 de abril de 2009 Aceptado: 23 de octubre de 2009

\title{
PRESENTACION
}

La institución educativa Limbania Velasco fue fundada por decreto departamental el 13 de febrero de 1906 con el nombre Escuela Colombia. Actualmente es una entidad departamental de carácter oficial con calendario A. Ofrece el ciclo educativo completo de educación básica y media desde el grado cero hasta quinto con jornada mañana y grado sexto a once en Jornada tarde. Su modalidad es académica con énfasis en competencias comunicativas. Atiende aproximadamente 700 estudiantes de ambos sexos de los estratos 1, 2, y 3, incluyendo población de las etnias indígena, negra y personas en situación de desplazamiento víctimas de la violencia armada.

La UNAD desde el año 2005 ha realizado un convenio interinstitucional de contraprestación el cual desde el programa de Psicología ha venido realizando intervención psicológica dirigida a 
los estudiantes. A través de este acercamiento y el diálogo con docentes surge la necesidad de abordar la convivencia escolar como objeto de estudio al observar en la población estudiantil bajo rendimiento escolar, indisciplina, agresiones y ausentismo injustificado. Además, los estudiantes poco acceden a las normas institucionales, les gusta violarlas constantemente y los padres de familia prestan poco apoyo e interés por el rendimiento escolar de sus hijos e hijas.

\section{FUNDAMENTACION TEÓRICA}

\section{Aproximación al concepto de convivencia escolar}

La convivencia, como su nombre lo indica, hace referencia a vivir con uno mismo y con los demás; tendencia natural del ser humano pero que se construye en las interacciones cotidianas de hombres y mujeres, su contexto geográfico y su cultura; implica aprender a convivir y a comunicarse. De allí que sea importante referenciar el siguiente concepto:

Convivir significa vivir unos con otros en base a unas determinadas relaciones sociales y a unos códigos, en el marco de un contexto social determinado donde los conflictos son inseparables por lo que no podemos pretender que las interacciones cotidianas y el proceso educativo transcurra sin conflictos, ni tampoco pensar que los mismos resultarán necesariamente negativos". ${ }^{4}$

Aprender a convivir consigo mismo, con los demás, con la naturaleza, con Dios y con las culturas es uno de los grandes retos de la sociedad actual al que se debe prestar mayor atención, esfuerzo y apoyo. En la educación del siglo xxI este es uno de los objetivos expresados por la UNESCO. Por ello, el principal ejercicio es implementar estrategias para el desarrollo de los aprendizajes básicos de una convivencia social. ${ }^{45}$ Aprendizajes donde se reconozca al otro como diferente más no como enemigo; se establezcan mecanismos adecuados de comunicación para afrontar positivamente el conflicto; se reconozcan sentimientos y se aprenda a compartir; aprendizajes para decidir en grupo, donde se promueva la participación democrática de las personas y se valore la diversidad de pensamiento; aprendizajes para cuidar tanto el bienestar físico como psicológico de sí mismo y de los demás; aprendizajes para cuidar el entorno donde se vive, donde se interactúa y donde se divierte; aprendizajes para valorar la diversidad étnica y cultural; fundamentos para la supervivencia y el desarrollo social - comunitario.

En el caso concreto de las instituciones educativas, dicho proceso supone un compromiso de todos los actores (directivos, docentes, padres, estudiantes), en la revisión de los conceptos de democracia, justicia, libertad, disciplina, autoridad, norma, conflicto, valores, entre otros; compromiso en el desarrollo de mejores interacciones y de la afectividad en la que se construyan relaciones de respeto y de cooperación a través de habilidades sociales y de aprendizaje

44 Rodríguez, Rodríguez. Rosabel. La acción tutorial para favorecer la convivencia: la disciplina. Universitat de les Illes Balears.

45 Siete Aprendizajes Básicos para la educación en la convivencia social de José Bernardo Toro A. Bogotá, Fundación social. 1992. 
cooperativo; compromiso con el desarrollo de competencias para la resolución adecuada del conflicto; compromiso con el ejercicio de democratizar los canales de comunicación para que ésta sea más dialógica, comprensible y conversacional en la práctica educativa y en el alcance de un aprendizaje satisfactorio.

Las instituciones educativas, después de la familia, se convierten en escenarios fundamentales para el aprendizaje de la convivencia; escenarios no solo para trasmitir conocimientos académicos sino para compartir con los otros, trabajar juntos, y ayudarse mutuamente. Es importante aprender a solucionar problemas, superar conflictos y aprender a vivir con las limitaciones y dificultades que cotidianamente ofrece la vida y el mundo que nos rodea.

Al entender la convivencia como el vivir en compañía, relacionarse e interactuar con los otros en un espacio determinado, la escolar se centra en las instituciones educativas las cuales establecen normas y códigos de interacción formales e informales para vivir armónicamente o hacer frente a los diversos conflictos de la vida humana. En la convivencia escolar se promueven relaciones autoritarias, sumisas, de exclusión, de diálogo, amor y respeto; incluye conceptos de disciplina, conflictos, valores, dignidad, justicia, solidaridad, derechos, deberes, ética y democracia. Desde este punto de vista, la convivencia escolar se relaciona con las vivencias e interacciones dadas entre los actores educativos y la comunidad estudiantil (administrativos, docentes, estudiantes y padres de familia) en un contexto determinado el cual promueve el desarrollo ético, socio afectivo e intelectual de los estudiantes.

En la comunidad educativa el tema de la convivencia escolar es fundamental no solo para la promoción de relaciones respetuosas y armoniosas sino para generar escenarios de participación, inclusión y reflexión en torno a los sistemas de comunicación, el manejo de las emociones, la tramitación justa del conflicto, la generación de espacios incluyentes y el ejercicio equitativo de los deberes y derechos humanos. Desde el punto de vista pedagógico Vargas, (2004), expresa: la comunidad educativa debe contribuir a la mayor participación en las actividades de la escuela y en los procesos educativos; al aprendizaje para la democracia, al desarrollo de habilidades para la convivencia; a la creación de un ambiente de estudio y de trabajo; y generar mayor integración de las relaciones entre profesores, estudiantes y padres de familia, entre otras.

\section{La normatividad escolar como un mecanismo de control}

Dentro de toda comunidad se establecen formas de organización, conocimientos, normas, valores, creencias y códigos de interacción, con el propósito de mantener una convivencia equilibrada y tranquila entre las personas que la componen; es por ello que dentro de un establecimiento educativo existe el reglamento de convivencia como mecanismo para enfrentar los diversos conflictos escolares y mantener una adecuada convivencia entre los actores educacionales. 
La normatividad escolar busca establecer mecanismos de convivencia en la escuela con el acompañamiento de los directivos, docentes, padres y estudiantes además de controlar las interacciones agresivas entre los actores educativos: la indisciplina y los conflictos al interior de las aulas, aunque no siempre es construida desde el consenso, sino que muchas están sustentadas desde una representación social hegemónica, a partir de los imaginarios del adulto, descontextualizada de la realidad social de los y las estudiantes.

Todos los establecimientos educativos la definen como un instrumento que rige las relaciones entre los miembros de la comunidad educativa, elaborado y entregado por la misma institución. Como reglamento debe ajustarse al marco legal entregado por la constitución nacional, a la convención de los derechos de los y las niños, los y las adolescentes, y la normativa dictada por el Ministerio de Educación Nacional (MEN). Además, debe considerar elementos de carácter formativo que permitan a los estudiantes comprender las normas, procedimientos, sanciones, derechos y deberes, que se establecen. Estas sanciones no pueden ser de carácter físico o que arriesguen las seguridades de niños, niñas y adolescentes, ni impedir el ingreso a la escuela. En este aspecto los consejos escolares tienen una oportunidad para participar en la revisión y elaboración de estos reglamentos en las comunidades escolares quienes establecen mecanismos adecuados y democráticos al interior de las instituciones educativas.

Una buena normatividad escolar posibilita mayor colaboración, resolución de conflictos y, lo más importante, una formación centrada en valores humanos que cada institución promueva y asegure la fundamentación de los estudiantes. Por otra parte, una buena normatividad escolar permite transmitir a los miembros de la comunidad educativa la idea de que los conflictos existen en forma permanente, pues basta que dos o más personas estén en desacuerdo respecto de algo para que éste surja. Por cierto, las emociones y sentimientos también tienen un rol protagónico en el conflicto. Saberlo, significa también entender que tras, el conflicto, la relación entre las partes puede quedar dañada o fortalecida y ello depende solo de la oportunidad y la forma en que haya abordado el problema.

Desde este punto de vista, la convivencia escolar se debe fundamentar en los siguientes principios normativos:

Principio de subordinación: sujeto a la ley colombiana y a las normas internacionales de protección humana ratificadas por el estado.

Principio de igualdad y no discriminación: al eliminar toda forma de arbitrariedad.

Principio de legalidad: al describir los comportamientos a sancionar y que las sanciones sean proporcionales a las faltas.

Principio de información: las normas deben ser conocidas por todos los miembros de la comunidad educativa.

Principio de formación: la norma debe tener un carácter formativo. 


\section{Los problemas de convivencia en las instituciones educativas}

Existe una preocupación creciente del profesorado y de la comunidad educativa respecto a los problemas de convivencia y disciplina que diariamente viven los centros educativos en donde se identifican agresiones físicas y verbales ya sea entre compañeros, agresiones dirigidas a las propiedades, exclusión social, falta de respeto hacia las normas o hacia el profesor, entre otros, y muchas vece no se atienden adecuada, ni oportunamente. Solo se les presta atención cuando son problemas graves o en el momento en el que suceden hecho que les resta importancia.

Recientemente se ha dado mayor atención a los problemas de convivencia escolar, tras la aparición de episodios violentos o dramáticos entre la comunidad educativa. Por ejemplo: maltrato a estudiantes, suicidio, racismo, muertes a estudiantes y docentes, vandalismo, acoso sexual, presencia de sustancias psicoactivas, armas, entre otros. Situaciones que angustian a los docentes, directivos y padres de familia y que también generan polémica acerca de los valores, la metodología de la enseñanza y la calidad de las instituciones educativas sin que se le preste una atención exhaustiva y prolongada.

Lo cierto es que los problemas de convivencia existen en diversas dimensiones con diferentes matices y en diferentes contextos ya sean relacionados con la agresividad (física y psicológica), con la indisciplina, con la tramitación inadecuada del conflicto, con comportamientos antisociales, con el no acatamiento a la norma o a la cultura ya sea porque la población escolar procede de contextos marginales, de violencia callejera, de violencia intrafamiliar, de marginalidad, por desplazamiento a causa del conflicto armado que actualmente vive nuestro país, entre otros. El problema está en cómo determinamos, clasificamos y atendemos los comportamientos inadecuados de los estudiantes dentro del centro escolar. ${ }^{46}$ De allí la importancia de comprender los problemas de convivencia además de desarrollar acciones psicosociales para el desarrollo de aprendizajes que mejoren las interacciones cotidianas al interior de las aulas escolares en las instituciones educativas.

\section{UN ENFOQUE PSICOSOCIAL PARA EL ABORDAJE DE LOS PROBLEMAS DE CONVIVENCIA ESCOLAR}

La intervención psicosocial es una disciplina de la psicología social que busca comprender y cambiar los procesos psicosociales para mejorar la calidad de vida de las personas. Este enfoque en el campo educativo se fundamenta en dos componentes clave. La calidad e interacción social donde se desarrollan las relaciones entre docentes - estudiantes, estudiantes - estudiantes, docentes - docentes, padres - hijos y las relaciones interpersonales dadas en los grupos al interior del aula escolar y los grupos informales. ${ }^{47}$

46 LUCA DE TENA DE BETHENCOURT, María del Carmen ; RODRÍGUEZ R, Rosa Isabel. La acción tutorial para favorecer la convivencia: la disciplina. Universitat de les Illes Balears. Palma de Mallorca, Conselleria de Educación y Cultura, 2001.

47 ÁlVARO. José Luis. GARIDO Alicia. OVEJERO Anastasio. Psicología social de la educación en Psicología Social Aplicada. J. R. Torregrosa. MCgrawllil. Interamericana de España, S.A. U. Madrid, 1999. 
Desde este enfoque y para el desarrollo de la investigación se tuvieron en cuenta las relaciones interpersonales e intergrupales dadas al interior de las aulas escolares de la población objeto de estudio para el análisis de los problemas de convivencia e indisciplina donde se desarrolla el proceso de enseñanza - aprendizaje, además de la normatividad escolar en el ejercicio de mantener y controlar el ambiente grupal y escolar.

\section{ENFOQUES PSICOSOCIALES SOBRE LAAGRESIVIDAD Y LA VIOLENCIA ESCOLAR}

Desde la psicología existen muchas teorías sobre las causas para explicar la agresión o la violencia. Unas se agrupan al vincular la agresión como una pulsión o fuerza interna de las personas y otras al explicarla desde los factores externos originados en el medio ambiente. Entre las teorías que se vinculan con el origen de la agresión desde los impulsos internos del ser humano se destacan la teoría de los instintos y las teorías biológicas; y desde las condiciones externas la teoría de la frustración - agresión y la teoría del aprendizaje social.

La teoría de los instintos en la que sobresalen dos autores; Sigmud Freud, ${ }^{48}$ quien expone que la agresión es un instinto destructivo y perturbador en la especie humana que requiere ser canalizado por estrategias no destructivas y Korad Lorenz ${ }^{49}$ quien dice que la agresión entre las espacies es más un recurso de adaptación esencial para la supervivencia, para la defensa, para la alimentación. Esta teoría ha sido criticada porque se ha podido probar que los diferentes actos agresivos son diversos en la forma de ejecutarlos, expresarlos y manifestarlos. Algunos personajes son violentos, otros no, unos usan armas, otros palabras y objetos, otros agraden activamente, otros de manera pasiva. ${ }^{50}$

Las teorías biológicas relacionan la agresión con las regiones cerebrales o del sistema nervioso, el cual influye en los impulsos emocionales y hormonales. Estos estudios tampoco se han logrado sustentar claramente debido a la influencia constante del medio ambiente en que se encuentre la persona.

La teoría de la frustración - agresión abordada por Jhon Dollard,$^{51}$ quien postula que la agresión es siempre consecuencia de la frustración y que la frustración siempre lleva alguna forma de agresión. ${ }^{52}$ Siendo la intensidad de la frustración la que determina la magnitud, tipología y los agentes de la agresión, esta teoría sostiene que la agresión es desplazada al agente frustrante. Además, se puede reducir por medio de la hipótesis de la Catarsis con el fin de minimizar los sentimientos de enojo, excitación, represión y privación de bienes, aspiraciones y libertades del individuo. Esta teoría también ha sido revisada porque la fuente de la agresión no siempre es la frustración, puede ser cualquier estimulo (instrumentos, necesidades, situaciones, personajes) del contexto. Más aún cuando el aprendizaje es fuente importante.

48 Médico y neurólogo austríaco fundador del psicoanálisis. (1856 - 1939).

49 Zoólogo austríaco galardonado con el Premio Nobel y fundador de la etolgía (1903 -1988)

50 UNAD. Módulo Psicología Social elaborado por Claudia Andrea Paredes. Bogotá. 2007. Pág. 151 - 152.

51 Psicoterapeuta estadounidense.

52 UNAD. Modulo Psicología Social elaborado por Claudia Andrea Paredes. Bogotá. 2007. Pág. 154 
La teoría del aprendizaje social propuesta por Albert Bandura ${ }^{53}$ y Wallers ${ }^{54}$ sostiene que en especial los niños aprenden cuándo, cómo, y a quién agredir. Sustentan cómo este aprendizaje se apoya en el reforzamiento y el modelamiento. El reforzamiento dado por la recompensa o elogios que el niño recibe de las relaciones con sus padres, amigos y del ambiente. El modelamiento se da cuando se imita el comportamiento de otros (padres, personales, héroes de acción) encontrados en la vivencia diaria o emitidos por los medios de comunicación. Esto nos indica que la agresión es una conducta aprendida a partir de la observación y que se puede modificar a partir de recompensas y modelos no agresivos como lo demostró Brown y Elliont (1965), a través del desarrollo de conductas prosociales que permitan disminuirla tales como la solidaridad, la cooperación, el altruismo. ${ }^{55}$

Este último modelo teórico es el que actualmente tiene mayor aceptación al incorporar factores cognitivos propios del sujeto y procesos simbólicos y auto regulatorios del medio. Las personas y en especial los niños y adolescentes adquieren representaciones de su realidad siendo estas guías para el comportamiento humano. Por ello ésta presta relevancia para la comprensión de los problemas de convivencia escolar y la promoción de aprendizajes cooperativos en la comunidad educativa.

Es importante mencionar que otro de los modelos que ha abordado la violencia es el modelo histórico desarrollado por Erich Fromm, ${ }^{56}$ quien distingue entre agresión benigna y agresión maligna. Maligna dada por una pulsión no orgánica que lleva a la destructividad y crueldad propia del hombre no heredada genéticamente; benigna dada por una pulsión orgánica necesaria para la supervivencia. Para Fromm los tipos de personalidad tienen que ver con pautas socioeconómicas concretas al considerar los seres humanos como producto de la cultura. De allí surge la necesidad de armonizar los impulsos del individuo y los de la sociedad donde vive. Desde este punto de vista, esta teoría también es útil para analizar los factores determinantes de los problemas de convivencia al interior de las aulas escolares y por qué no de la sociedad actual.

\section{DISEÑO METODOLÓGICO}

La metodología se fundamenta en la investigación acción IA, entendida como una estrategia que se enfoca en la búsqueda de alternativas de solución a la diversas problemáticas que vive la realidad educativa en torno a la convivencia escolar. Dentro del marco de la investigación cualitativa y desde la perspectiva de Alfonso Carrillo, ${ }^{57}$ ésta aborda en profundidad interacciones, experiencias, vivencias, sentimientos y pensamientos presentes en una

53 Psicólogo canadiense que hace referencia a las condiciones en que se aprende a imitar modelos.

54 Primer estudiante Graduado de Alber Bandura. Coautor del libro titulado: Agresión Adolescente en 1959.

55 MARTÍN. Baró. Ignacio. Acción e ideología. UCA Editores. El salvador. 1997.

56 Psicoloanalista germano estadounidense que aplicó la teoría psicoanalista a problemas sociales y culturales. $(1900-1980)$

57 TORRES CARRILLO, Alfonso. Aprender a investigar en comunidad II enfoques cualitativos y participativos en la comunicación social. Bogotá, facultad de ciencias sociales y humanas de Unisur, 1995. 
situación particular y en este caso permite describir la forma como estudiantes y docentes evidencian actitudes y prácticas cotidianas.

El grupo muestra para el desarrollo del trabajo se concentró en 79 estudiantes de los grados terceros de la jornada mañana (2 grupos) y 80 estudiantes de los grados octavos jornada tarde (3 grupos), correspondientes al 40\% de la población total de la institución educativa en el periodo escolar 2008. Se abordaron estrategias de investigación tales como la observación de aula, de recreo y de juego, la entrevista individual y grupal, la revisión de los observadores de cada estudiante, taller y las convivencias escolares con estudiantes. La investigación se desarrolló a través de las siguientes fases:

\section{Montaje institucional y Metodológico del proyecto}

Las acciones desarrolladas fueron la presentación del proyecto a docentes y directivos de la institución a través de reuniones, la selección y capacitación de un grupo de estudiantes de la UNAD como monitores para la consolidación de un equipo de trabajo. Se diseñaron los protocolos de observación, entrevistas, diarios de campo y revisión de documentos para la recolección de la información. Estos guiones y protocolos fueron puestos a prueba con un grado superior a la muestra en una jornada piloto que permitió validar contenidos y estrategias. En esta jornada se logró identificar cualidades y destrezas del equipo investigador en torno a los procesos de observación, comunicación, registro y pregunta de indagación.

\section{Descripción y caracterización de la vida cotidiana de la comunidad}

En esta fase se recolectó la información sobre las percepciones y prácticas institucionales en relación con las interacciones cotidianas, de convivencia y disciplina en las diferentes aulas escolares de los grados objeto de estudio se desarrollaron las siguientes estrategias según jornada académica:

Tabla 1. Estrategias según jornada académica

\begin{tabular}{|c|l|l|}
\hline JORNADA / GRADO & \multicolumn{1}{|c|}{ ESTRATEGIAS } & \multicolumn{1}{c|}{ PARTICIPANTES } \\
\hline \multirow{4}{*}{$\begin{array}{c}\text { MANANA GRADOS } \\
\text { TERCEROS } \\
\text { (2 grupos) }\end{array}$} & 2 Observaciones de aula & Estudiantes y docentes \\
\cline { 2 - 3 } & 1 Observación de recreo & Estudiantes en general \\
\cline { 2 - 3 } & 1 Observación de juego & Estudiantes del grado \\
\cline { 2 - 3 } & 1 Taller Yo y mis compañeros & Estudiantes de los grados 3-1 y 3-2 \\
\cline { 2 - 3 } & esperanza de vida & $\begin{array}{l}15 \text { Estudiantes con dificultades de } \\
\text { comportamiento y aprendizaje de } \\
\text { los grados 3-1 y 3-2 }\end{array}$ \\
\cline { 2 - 3 } & 1 entrevista grupal & $\begin{array}{l}2 \text { docentes directoras de grupo de } \\
\text { los grados 3-1 y 3-2 }\end{array}$ \\
\cline { 2 - 3 } & $\begin{array}{l}\text { Revisión de observador del } \\
\text { alumno }\end{array}$ & $\begin{array}{l}15 \text { estudiantes con dificultades de } \\
\text { comportamiento y aprendizaje }\end{array}$ \\
\hline
\end{tabular}




\begin{tabular}{|c|c|c|}
\hline \multirow{7}{*}{$\begin{array}{l}\text { TARDE GRADOS } \\
\text { OCTAVOS }\end{array}$} & 3 Observaciones de aula & $\begin{array}{l}\text { Estudiantes de los grados } 8-1.8-2 \\
\text { y } 8-3\end{array}$ \\
\hline & 1 Observación de recreo & Estudiantes en general \\
\hline & 1 Observación de juego & Estudiantes del grado 8-3 \\
\hline & 2 entrevistas individuales & $\begin{array}{l}2 \text { docentes directores de grupo de } \\
\text { los grados } 8-2 \text { y } 8-3\end{array}$ \\
\hline & Entrevista individual & $\begin{array}{l}8 \text { estudiantes con problemas de } \\
\text { comportamiento y aprendizaje de } \\
\text { los grados } 8-1,8-2 \text { y } 8-3\end{array}$ \\
\hline & Entrevista grupal & $\begin{array}{l}5 \text { lideres estudiantes de los grados } \\
8 \text {-1 y } 8-2 \text { (principal y suplente) }\end{array}$ \\
\hline & $\begin{array}{l}\text { Revisión del observador del } \\
\text { alumno }\end{array}$ & Estudiantes de los grados $8-2$ y $8-3$ \\
\hline
\end{tabular}

\section{Análisis de la convivencia y la disciplina en el contexto escolar}

La información recolectada en la fase anterior se tabuló de manera cualitativa a través de tablas y matrices de categorías y se analizó con el fin de describir e interpretar la realidad en torno a la convivencia escolar. Se realizó un taller con los estudiantes de los grados octavos con el fin de socializar y poner en común los problemas de convivencia encontrados al interior de las aulas escolares.

Definición de núcleos problemáticos y prácticas culturales dinamizadoras de la convivencia institucional. Esta fase consistió en realizar una síntesis de la realidad y las prácticas institucionales a través de mapas de conocimiento sobre los problemas encontrados y la ruta institucional de atención abordado mediante encuentros y grupos de discusión con docentes y líderes estudiantiles. Esta fase permitió identificar y definir estrategias para el desarrollo de aprendizajes sobre sí mismos, interacción, comunicación, valores, tratamiento del conflicto y proyectos de vida.

Puesta en marcha de plan de acción institucional: esta corresponde a la segunda parte del proyecto apenas en ejecución. Consiste en desarrollar diez talleres con los estudiantes de ambas jornadas a fin de promover aprendizajes de reconocimiento y convivencia. Se pretende que a partir de las estrategias de acción, se tomen decisiones frente al abordaje de los conflictos y así transformar la cultura escolar. En esta fase se combinan diferentes técnicas como talleres, convivencias, actividades lúdicas, entre otras.

Evaluación y sistematización del proceso: las acciones pertinentes a esta fase se incluyeron en cada una de las anteriores etapas del trabajo. Se organiza la información recopilada y los talleres se registran a través de cartillas educativas. 


\section{RESULTADOS Y DISCUSIÓN}

\section{PERCEPCIONES SOBRE LA CONVIVENCIA ESCOLAR}

Los resultados se presentan considerando unidades de análisis y categorías de las entrevistas que se realizaron con docentes y estudiantes sobre sus significados expresados en relación con sus vivencias y conocimientos.

Tabla 2. Percepciones del docente sobre el clima escolar

\begin{tabular}{|c|c|}
\hline CATEGORÍA & INDICADOR \\
\hline \multirow[t]{5}{*}{ Relaciones docente - estudiante } & Con rigidez y autoritarismo \\
\hline & Poca comprensión \\
\hline & Son excelentes \\
\hline & Trato como iguales \\
\hline & Algunos maestros son conciliadores y comprensivos \\
\hline $\begin{array}{l}\text { Contenidos de diálogo docentes - } \\
\text { estudiantes }\end{array}$ & $\begin{array}{l}\text { Gustos, realidades, problemas personales, familiares } \\
\text { y del país }\end{array}$ \\
\hline Relaciones docente - docente & Excelentes con algunas asperezas \\
\hline \multirow[t]{5}{*}{ Sentimientos de los estudiantes } & Requiere ser aconsejado y escuchado \\
\hline & Se muestran alegres, con energía \\
\hline & Son francos y expresan lo que piensan \\
\hline & Pocos se muestran tristes \\
\hline & Algunos reflejan a sus padres \\
\hline \multirow[t]{2}{*}{ Sentimientos de los docentes } & Requieren mentalidad joven \\
\hline & $\begin{array}{l}\text { Requieren estar equilibrados para compartir sus } \\
\text { experiencias y conocimientos }\end{array}$ \\
\hline \multirow{2}{*}{$\begin{array}{l}\text { Sentimientos de los padres de } \\
\text { familia }\end{array}$} & Algunos son corteses y joviales \\
\hline & Algunos son amargados y groseros \\
\hline \multirow[t]{2}{*}{ Problemas vividos } & $\begin{array}{l}\text { El 100\% de los estudiantes no logran buen rendimiento } \\
\text { escolar }\end{array}$ \\
\hline & $\begin{array}{l}\text { Los estudiantes no tienen las mismas inteligencias, } \\
\text { deseos ni gustos }\end{array}$ \\
\hline
\end{tabular}


Tabla 3. Percepción de los estudiantes sobre el contexto escolar. Parte 1

\begin{tabular}{|c|c|c|}
\hline CATEGORÍA & LAS ADOLESCENTES & LOS ADOLESCENTES \\
\hline $\begin{array}{l}\text { Representación de la palabra } \\
\text { [Escuela] o [colegio] }\end{array}$ & $\begin{array}{l}\text { Estudio - amigos - conocimiento } \\
\text { - aprendizaje - Estudiantes y } \\
\text { profesores }\end{array}$ & $\begin{array}{l}\text { Profesores y estudiantes } \\
\text { Conocimiento - Pereza }\end{array}$ \\
\hline Importancia del aprender & $\begin{array}{l}\text { Mucho - Sin conocimiento uno no } \\
\text { es nada - Tener conocimiento - Ser } \\
\text { alguien en el futuro - Ser alguien } \\
\text { en la vida }\end{array}$ & $\begin{array}{l}\text { Para ser alguien en la vida } \\
\text { - Quiero aprender - Si uno } \\
\text { aprende puede capacitarse }\end{array}$ \\
\hline Gustos & $\begin{array}{l}\text { Hacer trabajos para despejar } \\
\text { la mente - Compartir con mis } \\
\text { compañeros - Algunos profesores } \\
\text { - Es grande y hay ambiente }\end{array}$ & $\begin{array}{l}\text { Aprender y estar con los amigos } \\
\text { - Me gusta la música y el teatro - } \\
\text { Me gusta cuando entiendo - los } \\
\text { profesores cuando explican }\end{array}$ \\
\hline Proyecto de vida & $\begin{array}{l}\text { Estudiar criminalística - Ser } \\
\text { comunicadora social - Ser doctora } \\
\text { - Estudiar medicina - Enfermería }\end{array}$ & $\begin{array}{l}\text { Trabajar en la Marina - Estudiar } \\
\text { electricidad - Trabajar en una } \\
\text { óptica }\end{array}$ \\
\hline Apoyo escolar & $\begin{array}{l}\text { Con algunas materias - Ninguno } \\
- \text { Casi no }\end{array}$ & $\begin{array}{l}\text { Consejos } \quad-\quad \text { Aprendizaje- } \\
\text { Ninguno- En parte si }\end{array}$ \\
\hline Relación entre compañeros & $\begin{array}{l}\text { Algunos son altaneros - Bien } \\
\text { Algunos son muy cansones }\end{array}$ & $\begin{array}{l}\text { Buenas y amigables - Bien - } \\
\text { Con la mayoría hablo }\end{array}$ \\
\hline $\begin{array}{l}\text { Percepción sobre el rol del } \\
\text { maestro }\end{array}$ & $\begin{array}{l}\text { Nos enseñan valores - Enseñan lo } \\
\text { normal - Nos colocan malas notas } \\
\text { - Cuando nos portamos mal - Nos } \\
\text { orientan - Dialogan y nos regañan }\end{array}$ & $\begin{array}{l}\text { Los maestros nos enseñan y } \\
\text { aconsejan, nos hacen salidas } \\
\text { de integración. Deben ser más } \\
\text { estrictos- Nos regañan - Que } \\
\text { estudien bien los temas }\end{array}$ \\
\hline
\end{tabular}

Tabla 3. Percepción de los estudiantes sobre el contexto escolar. Parte 2

\begin{tabular}{|c|c|c|}
\hline CATEGORÍA & LAS ADOLESCENTES & LOS ADOLESCENTES \\
\hline $\begin{array}{l}\text { Problemas de indisciplina } \\
\text { en el aula }\end{array}$ & $\begin{array}{l}\text { Mucha recocha - Todos los días } \\
\text { se pierde algo en el salón - El no } \\
\text { respeto - La bulla - Desorden }\end{array}$ & $\begin{array}{l}\text { Molestar y hablar en clases - Colocar } \\
\text { sobrenombres - No poner atención }\end{array}$ \\
\hline $\begin{array}{l}\text { Participación en el cuidado } \\
\text { de la I. E. }\end{array}$ & $\begin{array}{l}\text { Ninguna } \\
\text { Con el aseo }\end{array}$ & Ninguna \\
\hline Materias de preferencia & $\begin{array}{l}\text { Ciencias naturales - Biología Ética } \\
\text { - Castellano }\end{array}$ & Inglés - álgebra - Español - ingles \\
\hline $\begin{array}{lll}\text { Materias de } & \text { poca } \\
\text { preferencia } & & \\
\end{array}$ & $\begin{array}{l}\text { Inglés - álgebra - Geometría - } \\
\text { español }\end{array}$ & Sociales- Álgebra \\
\hline $\begin{array}{lcc}\text { Percepción } & \text { frente } \\
\text { rendimiento académico } & \end{array}$ & $\begin{array}{l}\text { Me va regular - Pierdo materias } \\
\text { Pésimo - Sobresaliente pero quiero } \\
\text { mejorar - bien pero pierdo algebra }\end{array}$ & $\begin{array}{l}\text { Es regular. No hago tareas. Me da } \\
\text { pereza - Bueno }\end{array}$ \\
\hline
\end{tabular}




\begin{tabular}{|c|c|c|}
\hline Dificultades escolares & $\begin{array}{l}\text { Pienso en mi y en todo - Falta más } \\
\text { relación con mi mamá - Llega } \\
\text { de mal genio y a veces me pega - } \\
\text { Trato con los compañeros hombres } \\
\text { - Algunas cosas no entiendo - Me } \\
\text { distraigo mucho- No pongo atención } \\
\text { a los profesores }\end{array}$ & $\begin{array}{l}\text { Por el cabello largo - No llevarse } \\
\text { bien con algunos profesores - La } \\
\text { lejanía }\end{array}$ \\
\hline $\begin{array}{l}\text { Representación de la } \\
\text { palabra [Convivencia] }\end{array}$ & $\begin{array}{l}\text { Relación con otras personas - No es } \\
\text { buena en el colegio - Compartir - Ser } \\
\text { amigable - En el colegio es vacana - } \\
\text { Compartir -En el salón es regular } \\
\text { y en el colegio mala - Es buena - } \\
\text { Compartir unos con otros }\end{array}$ & $\begin{array}{l}\text { Relación con las personas - En el } \\
\text { colegio es buena - Saber tratar con } \\
\text { los demás - En el colegio es buena - } \\
\text { Se ven peleas }\end{array}$ \\
\hline $\begin{array}{l}\text { Representación de la } \\
\text { palabra [Disciplina] }\end{array}$ & $\begin{array}{l}\text { Comportarse bien - Nada buena en el } \\
\text { colegio - Buen comportamiento } \\
\text { No hay respeto - Orden en todo - En } \\
\text { el colegio es regular }\end{array}$ & $\begin{array}{l}\text { Comportamiento - En el salón es } \\
\text { regular - juicio. En el colegio es un } \\
\text { poco mala - Son desobedientes }\end{array}$ \\
\hline Comentarios adicionales & $\begin{array}{l}\text { Que cambien el uniforme. No me } \\
\text { gusta la pechera. }\end{array}$ & El colegio es bueno - Es muy suave \\
\hline
\end{tabular}

Tabla 4. Presencia del conflicto en las aulas escolares

\begin{tabular}{|l|l|}
\hline CATEGORÍA & \multicolumn{1}{|c|}{ INDICADOR } \\
\hline \multirow{4}{*}{ Tipología } & Por las maneras de ser, pensar y actuar \\
\cline { 2 - 2 } & Apodos y discusiones \\
\cline { 2 - 2 } & Por el aseo \\
\cline { 2 - 2 } & Conductas agresivas \\
\hline Involucrados & Padres y docentes \\
\cline { 2 - 2 } & Los mismos estudiantes \\
\hline \multirow{3}{*}{ Tramitación } & El dialogo entre las partes \\
\cline { 2 - 2 } & El maestro como mediador \\
\cline { 2 - 2 } & Los representantes estudiantiles pueden contribuir \\
\cline { 2 - 2 } & Los representantes estudiantiles poco asumen su rol \\
\hline
\end{tabular}


Tabla 5. Valoración y protección de los y las adolescentes

\begin{tabular}{|l|l|}
\hline \multicolumn{1}{|c|}{ CATEGORÍA } & \multicolumn{1}{c|}{ INDICADOR } \\
\hline Importancia & $\begin{array}{l}* * \text { Los estudiantes son la razón de ser del docente. } \\
* * \text { De la formación del estudiante depende su futuro, la convivencia } \\
\text { y el desarrollo del país. }\end{array}$ \\
\hline Expresión de cariño & $* *$ Expresiones de afecto $* *$ Buen trato \\
\hline $\begin{array}{l}\text { Expresión de los } \\
\text { adolescentes }\end{array}$ & $\begin{array}{l}* * \text { Expresiones según la personalidad extrovertido e introvertido } \\
\text { Expresión según afecto y comportamiento }\end{array}$ \\
\hline Riesgos & $* *$ Alto consumo y expendio de la droga en el municipio \\
\hline Tipo de violencia & $\begin{array}{l}* * \text { Agresividad verbal } \\
* * \text { Discriminación racial y étnica entre compañeros }\end{array}$ \\
\hline Fuentes de protección & $* *$ Instituciones de atención y protección estatal: Hospital, ICBF, UNAD \\
\hline Tipo de tareas asignadas & $* *$ Responsabilidades escolares \\
& $* *$ Mandados del docente \\
& $* *$ El aseo escolar \\
\hline
\end{tabular}

Tabla 6. Bienestar de los niños y niñas vulnerables al interior de la institución

\begin{tabular}{|l|l|}
\hline \multicolumn{1}{|c|}{ CATEGORÍA } & \multicolumn{1}{c|}{ INDICADOR } \\
\hline Niños - jóvenes vulnerados & $\begin{array}{l}\text { Niños desplazados } \\
\text { Niños con problemas de aprendizaje } \\
\text { Niños con problemas de atención }\end{array}$ \\
\hline Estrategias de atención & $\begin{array}{l}\text { Atención equitativa } \\
\text { Se identifica el problema en el aula } \\
\text { Se remite a un profesional (psicólogo }- \text { otro) }\end{array}$ \\
\hline
\end{tabular}

Tabla 7. Participación de los y las adolescentes en el contexto escolar

\begin{tabular}{|c|ll|}
\hline CATEGORÍA & \multicolumn{1}{|c|}{ INDICADOR } \\
\hline Participación Escolar & $* *$ & Participación en el aula escolar \\
& $* *$ & Participación en grupos culturales \\
& $* *$ & Participación en deporte \\
& $* *$ & Participación en eventos institucionales (Evento de \\
& & la creatividad - Feria del arte y la cultura) \\
\hline
\end{tabular}


Tabla 8. Manual de convivencia escolar

\begin{tabular}{|c|c|}
\hline CATEGORÍA & INDICADOR \\
\hline Tiempo y modo de construcción & $\begin{array}{l}\text { ** } 4 \text { años de construcción. } \\
\text { * Se ha construido por fases. } \\
\text { ** Se construye de acuerdo a las necesidades. } \\
\text { ** Se busca que todos participen }\end{array}$ \\
\hline Participantes & $\begin{array}{l}* * \text { Estudiantes } \\
* * \text { Docentes } \\
* * \text { Padres de Familia }\end{array}$ \\
\hline Difusión / socialización & $\begin{array}{l}\text { ** Adquisión por compra directa } \\
\text { ** Trabajo desde las áreas de sociales y ética. } \\
\text { ** Discusión en el aula en direcciones de grupo }\end{array}$ \\
\hline Participación / discusión & $\begin{array}{l}\text { ** Unidad del plan de estudios } \\
* * \text { Aula escolar desde las aéreas de estudio }\end{array}$ \\
\hline Aplicabilidad & $\begin{array}{l}\text { ** No se da por desconocimiento del manual } \\
* * \text { Se conoce por obligación o por una nota } \\
* * \text { No hay un habito de lectura institucional } \\
* * \text { Es viable y aplicable }\end{array}$ \\
\hline
\end{tabular}

Tabla 9. Sentido de vida y visión de futuro del docente

\begin{tabular}{|c|c|}
\hline CATEGORÍA & INDICADOR \\
\hline Como se siente & $\begin{array}{l}* * \text { Bien. } \\
* * \text { He tenido vocación } \\
\text { ** No he tenido conflictos ni inconvenientes } \\
\text { ** Uno se cansa } \\
\text { ** Tengo sueños por cumplir }\end{array}$ \\
\hline Proyecto de vida & $\begin{array}{l}* * \text { Escribir } \\
* * \text { Ser docente investigadora } \\
\text { ** Trabajar en una universidad } \\
\text { ** Trabajar en el extranjero } \\
\text { ** Estar mejor }\end{array}$ \\
\hline $\begin{array}{l}\text { Estrategias de apoyo } \\
\text { institucional }\end{array}$ & $\begin{array}{l}\text { ** Presupuesto precario para fotocopias y proyectos } \\
\text { ** Se requiere gestión } \\
\text { ** Se han logrado algunas cosas } \\
\text { ** Yo me ayudo personalmente en mejorar } \\
\text { ** Participación en convocatorias y capacitaciones }\end{array}$ \\
\hline
\end{tabular}


Tabla 10. Identidad personal de los estudiantes

\begin{tabular}{|c|c|c|}
\hline CATEGORÍA & LAS ADOLESCENTES & LOS ADOLESCENTES \\
\hline Gustos de vida & $\begin{array}{l}\text { No se - La rumba - Estudiar - La } \\
\text { juventud }\end{array}$ & $\begin{array}{l}\text { Mi familia porque los quiero mucho } \\
\text { - Estudiar - Mi familia porque la } \\
\text { tengo a mi lado }\end{array}$ \\
\hline Disgustos de vida & $\begin{array}{l}\text { No me gusta nada - Los problemas: } \\
\text { La entrega de boletas a mi mamá - } \\
\text { Hacer oficio - La separación de mis } \\
\text { padres }\end{array}$ & $\begin{array}{l}\text { Los problemas en el colegio - Los } \\
\text { castigos por perdida de materias }\end{array}$ \\
\hline Sentimiento de Alegría & $\begin{array}{l}\text { Cuando estoy con mis amigos de } \\
\text { salón - La recocha; Soy loca; Me } \\
\text { gusta ser popular - Sacar buenas } \\
\text { notas - Estar con mi familia - Visitar } \\
\text { a mi familia }\end{array}$ & $\begin{array}{l}\text { Mis amigos - Estar con mis } \\
\text { compañeros de clase - cuando salgo } \\
\text { a la calle y escucho música }\end{array}$ \\
\hline Sentimiento de Tristeza & $\begin{array}{l}\text { Cuando amigos o familia le fallen o } \\
\text { nos den la espalda - Que mis padres } \\
\text { no estén juntos - Perder materias } \\
\text {-Cuando me regañan }\end{array}$ & $\begin{array}{l}\text { Los recuerdos de un primo fallecido- } \\
\text { No Tener papá - cuando le pegan a } \\
\text { los perros }\end{array}$ \\
\hline Sentimiento de Enojo & $\begin{array}{l}\text { Que no me digan las cosas en la cara } \\
\text { - El desorden me estresa - Cuando } \\
\text { me regañan - Que me excluyan } \\
\text { del grupo de trabajo - Cuando me } \\
\text { preguntan y no se }\end{array}$ & $\begin{array}{l}\text { Cuando me mandan hacer cosas que } \\
\text { no quiero - Hacer las cosas con } \\
\text { presión - Cuando pierdo materias }\end{array}$ \\
\hline La mayor parte del tiempo la paso & $\begin{array}{l}\text { Triste y sola - De tres ánimos } \\
\text { desde la separación de mis padres } \\
\text {-Contenta -Alegre }\end{array}$ & Contento \\
\hline Me hace falta para ser feliz & $\begin{array}{l}\text { Mi abuelo y mi mamá } \\
\text { Mi padres juntos }\end{array}$ & Las mujeres - Mas libertad \\
\hline
\end{tabular}

Estas descripciones indican que en la jornada tarde la percepción sobre la convivencia escolar es representada por una relación, una actitud o una condición del ser humano para acercarse a los otros e interactuar en un contexto determinado. Se representa como algo complejo y diverso al vincularse las relaciones familiares y sociales del mundo en general. En los estudiantes no se evidencia claramente si es positiva o negativa, cada uno la califica según sus vivencias y experiencias. Los docentes a nivel general la expresan como positiva aunque se identifican comportamientos inadecuados y conflictos que requieren ser atendidos.

En la jornada mañana docentes y estudiantes representan la convivencia. Es un espacio propicio para fomentar los valores y el buen trato dentro y fuera del aula. Las niñas opinan al respecto: “... la convivencia minimiza los conflictos, y los rencores en el aula de clase y nos ayuda a fortalecer los procesos de aprendizaje”. La convivencia se define como una herramienta clave para transformar los comportamientos y las actitudes de los estudiantes en este caso niños y niñas. Por medio de la convivencia los estudiantes reflexionan y manejan mejor sus emociones cuando se presentan ante diversas situaciones nuevas o conflictivas. 


\section{PRÁCTICAS QUE OBSTACULIZAN Y POSIBILITAN LA CONVIVENCIA ESCOLAR}

\section{Una mirada al contexto escolar de la jornada mañana}

Desde este contexto se encuentran las siguientes prácticas que obstaculizan la convivencia escolar:

Los padres de familia son ajenos a las problemáticas escolares, el encuentro de padres solo se da en la entrega de boletines, a cada cual solo le interesa su hijo, no hay un apoyo colectivo a la problemática social de la institución por parte de las familias. Por otro lado, los estudiantes no acatan normas, se levantan cada momento del puesto. Esto genera indisciplina y distracción a los compañeros que están atentos a la clase. Cuando la profesora se retira por un momento del aula de clase los estudiantes hablan en voz alta, no controlan emociones, se empujan, arrastran los pupitres, no existe un auto control. Además, en los hogares hay muy poca comunicación con los hijos, los padres no ejercen autoridad de una manera adecuada, algunos son permisivos y otros autoritarios. Esto hace que se evidencie poco diálogo, y no se desarrolle una comunicación asertiva por parte de los padres y adultos. Otro aspecto a resaltar en las familias es la constante presencia de conflictos que al no ser abordados de una manera adecuada afectan la estabilidad emocional de los niños y niñas y por ende su bajo rendimiento académico.

Los conflictos en las relaciones familiares son motivo de inestabilidad emocional el que afecta el bajo rendimiento académico en los estudiantes. Esto se evidencia en la falta de interés por el estudio, desgano, indisciplina y mal comportamiento dentro y fuera del aula. Además los estudiantes realizan actos agresivos hacia los demás compañeros ampliando el círculo de violencia.

Entre las estrategias que posibilitan de una manera adecuada las interacciones cotidianas se encuentran las siguientes: Las profesoras establecen espacios de diálogo para escuchar a los niños y niñas, y les brindan apoyo emocional cuando lo requieren, no solo en aula sino en el patio de recreo; la orientación y el desarrollo de talleres a los cuales ellos responden complacidos, escuchan y participan activamente. En el aula escolar la comunicación entre compañeros ellos la califican como buena, especialmente con el grupo de amigos que escogen, igual el diálogo con el profesor. Las profesoras ejercen su labor de líder con frases que tienden a cambiar la conducta negativa del alumno de modo aceptable. Corrigen su vocabulario, establecen normas que el estudiante debe cumplir, como el ingreso a tiempo después del timbre.

\section{Una mirada al contexto escolar de la jornada tarde}

Las prácticas educativas se describen a partir de la interacciones interpersonales (docenteestudiante; estudiante - estudiante; docente - docente), los comportamientos individuales y grupales, y el trato entre las personas. A nivel institucional se encuentran muchas de las prácticas docentes y cotidianas que privilegian el autoritarismo, la exclusión y la sumisión, como también otras que dan cabida a relaciones afectuosas, humanas, abiertas al diálogo y a 
la solidaridad. La problematización de estas prácticas de convivencia escolar, permitieron reconocer sus limitaciones, posibilidades y propuestas para su transformación.

En cuanto a las interacciones personales se destacan acciones autoritarias y excluyentes que deterioran la convivencia entre ellas se identifican los gritos, las palabras rígidas, los reportes disciplinarios, la poca comprensión del docente, las agresiones verbales y físicas entre estudiantes, la presencia de objetos que interfieren en las aulas escolares como el celular, maquillaje, radios, etc. Entre las que se destacan como positivas están la comprensión, la escucha, la atención oportuna, la cortesía, el trato igual entre pares, la conciliación, el diálogo sobre aspectos personales, académicos y familiares, la consejería dada del docente al estudiante. Las relaciones establecidas entre los actores algunos las califican como excelentes otras como deterioradas. Anivel institucional se encontró, entre otras, las izadas de bandera, la participación en eventos culturales tales como la feria de arte y de la cultura, las salidas de campo, una comida comunitaria, las integraciones y paseos institucionales, campeonatos deportivos, jean days, reinados y bailes de despedida como acciones que favorecen la convivencia además hacen parte de la cultura escolar como tal. Estas permiten el reconocimiento del otro, su inclusión, participación y ayuda mutua.

Los comportamientos individuales y grupales se definen como adecuados e inadecuados. Entre los adecuados se destacan los que contribuyen al desarrollo de la armonía y el cumplimiento de la normatividad escolar establecida. En este caso se refiere a ser responsables con el trabajo en el caso de los docentes y las tareas académicas en el caso de los estudiantes, portar el uniforme adecuadamente, ingresar al aula de clase en el momento indicado o cuando suena el timbre, buena actitud para el trabajo académico, estar dispuestos para la participación en clases, higiene personal, orden y limpieza en el aula de clases, el cumplimiento de horarios establecidos. En conclusión, buena postura, disciplina y actitud personal y grupal.

Los comportamientos inadecuados son aquellos que se encuentran al margen de la normatividad y no están socialmente aceptados. Se relaciona con los actos de indisciplina manifestados de diferentes formas que perturba la convivencia armónica y el clima escolar además constantemente se convierten en un problema o conflicto, en este caso escolar. Aquí se identifican la amenaza, insultos, los apodos y molestias personales, agresiones físicas y verbales, la indiferencia, robos de objetos escolares o dinero, la presencia de armas o juegos bélicos en la institución, la presencia de sustancias psicoactivas, hablar en horas de clase o cuando alguien está hablando, la poca concentración y atención en las clases o actividades académicas, la recocha y la rebeldía de algunos adolescentes.

El trato personal en algunas ocasiones se evidencia de una manera afectuosa y en otras de manera agresiva e intolerante. Se dan de cuerdo al estado de ánimo y al manejo de las emociones personales. El trato afectuoso se da en varias ocasiones cuando los comportamientos son adecuados y se cumple con la normatividad escolar, además cuando se manifiestan personalidades alegres, cariñosas e introvertidas. Agresivo cuando no se presta atención y se encuentran sentimientos de rebeldía, enojo, desobediencia y disgustos personales. 


\section{PROBLEMAS O CONFLICTOS CENTRALES EN LA CONVIVENCIA ESCOLAR DE LA COMUNIDAD EDUCATIVA LIMBANIA VELASCO}

Los problemas de convivencia se relacionan con los comportamientos inadecuados, problemas de disciplina y la presencia de conflictos no solo en el contexto escolar sino también personal, familiar y social. Entre ellos se destacan los siguientes:

Tabla 11. Clasificación de los problemas encontrados

\begin{tabular}{|l|l|l|l|}
\hline \multicolumn{1}{|c|}{ PERSONALES } & \multicolumn{1}{|c|}{ FAMILIARES } & \multicolumn{1}{c|}{ ESCOLARES } & \multicolumn{1}{c|}{ SOCIALES } \\
\hline Poco liderazgo del estudiante & Manejo inadecuado del conflicto & Normas inadecuadas & Consumo y expendio de droga \\
Rebeldía y enojo de los estudiantes & Trato inadecuado & Manejo inadecuado del conflicto & Discriminación racial y \\
Conductas agresivas & Poca espiritualidad & Incumplimiento de las normas de & étnica entre compañeros \\
Personalidad agresiva & Imposición de normas & disciplina & \\
Problemas de aprendizaje & Deficiente comunicación & Bajo rendimiento académico & \\
Problemas de concentración & Inadecuadas relaciones interpersonales & Poca comprensión docente de los & \\
Problemas de atención & Discusiones & Rigidez y autoritarismo de loces & \\
Bajo rendimiento escolar & Apodos & docentes & \\
Agresividad verbal & & Trato inadecuado entre compañeros & \\
Necesidad de afecto, amor y cariño & & Imposición de normas & \\
Desinterés por el aprendizaje & & Poca o nula aceptación de normas & \\
& & Poca concentración en el aula & \\
& & Inadecuadas relaciones interpersonales & \\
& & Discusiones & \\
& & Presencia de objetos que interfieren & \\
& & el aula escolar & \\
& & Discriminación racial y étnica & \\
& & entre compañeros & \\
& & Apodos & \\
\hline
\end{tabular}

Tabla 12. Problemas escolares, factores y abordaje

\begin{tabular}{|c|c|c|}
\hline FACTORES QUE INCIDEN & $\begin{array}{l}\text { PROBLEMAS DE CONVIVENCIA } \\
\text { ESCOLAR }\end{array}$ & ABORDAJE INSTITUCIONAL \\
\hline $\begin{array}{l}\text { Los problemas del hogar } \\
\text { El trato inadecuado de padres y docentes } \\
\text { La problemática social: Narcotráfico } \\
\text { Desconocimiento del manual de } \\
\text { convivencia } \\
\text { La poca responsabilidad asignada } \\
\text { Poca comprensión de las orientaciones } \\
\text { y en la aplicación de la norma } \\
\text { La rigidez y el autoritarismo de los } \\
\text { docentes } \\
\text { Trato inadecuado entre iguales } \\
\text { El estudiante le gusta llamar la } \\
\text { atención. }\end{array}$ & $\begin{array}{l}\text { Prácticas y relaciones agresivas entre pares } \\
\text { Agresión verbal } \\
\text { Presencia de objetos que interfieren en el aula } \\
\text { escolar (celulares, maquillaje, ruido) } \\
\text { Incumplimiento de las normas de disciplina } \\
\text { Riñas y alegatos entre algunos estudiantes } \\
\text { Poca concentración de los estudiantes en } \\
\text { las aulas escolares }\end{array}$ & $\begin{array}{l}\text { Actividades de integración escolar } \\
\text { El diálogo entre docentes y estudiantes } \\
\text { Establecimiento de reglas de trabajo } \\
\text { Apoyo profesional (psicología) } \\
\text { Las direcciones de grupo } \\
\text { Reflexiones grupales } \\
\text { Conducto regular desde el Manual } \\
\text { de convivencia. } \\
\text { Establecer un plan de acción insti- } \\
\text { tucional } \\
\text { El diálogo y comprensión del docente } \\
\text { La identificación de los problemas }\end{array}$ \\
\hline
\end{tabular}

Al realizar una lectura de las descripciones anteriores se refleja cómo los estudiantes muchas veces toman la justicia por cuenta propia, al ejercer la violencia como una forma de defenderse y liberar sus sentimientos reprimidos. La agresividad y la violencia se ven reflejados en el sistema de comunicación, en las relaciones interpersonales, la intolerancia y el irrespeto frente a las opiniones del otro, en el vocabulario inapropiado al dirigirse a sus compañeros como al docente, los juegos bruscos y la presencia de riñas por diversos motivos, la actitud desafiante y rebelde de algunos estudiantes frente a los llamados de atención no solo de sus maestros 
sino de sus padres de familia. Es importante mencionar que los padres de familia y algunos docentes también manifiestan rasgos de agresividad y violencia siendo un mal ejemplo de autoridad y disciplina. Estas respuestas refuerzan la baja autoestima y genera tendencias auto agresivas buscando captar la atención y ocultar dificultades personales.

La influencia de la vida cotidiana y familiar valida la ofensa, la burla, los apodos, el golpe, el menosprecio, el maltrato y la exclusión como también el consumo y el expendio de sustancias psicoactivas influyen de una manera determinada en las prácticas y relaciones humanas expresadas en el aula escolar y en los juegos cotidianos. Se evidencia ausencia total de los estudiantes en la construcción de normas de convivencia. Entre los mecanismos pacíficos para la resolución de conflictos se encuentran el diálogo y la mediación del docente pero es necesario desarrollar espacios que permitan el empoderamiento de los líderes estudiantiles, su capacidad de liderazgo, el ejercicio de la autonomía y la responsabilidad de cada estudiante.

Tal como lo menciona Garnica Moreno y sus colaboradores (2005), es importante hacer una diferencia entre los actos de violencia y los actos de indisciplina. Los actos de violencia surgen a partir de los procesos subjetivos, de las interrelaciones personales, que se nutren de las representaciones y significaciones imaginarias de cada sujeto psíquico desencadenadas en el contexto escolar; y los actos de indisciplina aunque se refieren al sujeto y a sus relaciones intersubjetivas, estas se fundan en las relaciones pedagógicas dadas en el aula o en la institución. Dichos actos se relaciona, además, con los modos de enseñar, el uso de espacios, tiempos y normas institucionales. Se trata más bien de comportamientos que los estudiantes realizan dificultando o impidiendo el desarrollo de una clase o actividad normal, molestando u hostigando a sus compañeros que no necesariamente son hechos de violencia y agresividad. Esto remite la necesidad de revisar o cuestionar la práctica pedagógica y la función del docente.

Otro aspecto fundamental de análisis es el conflicto que no necesariamente debe ser abordado con la violencia. El conflicto es inherente a la vida humana, se presenta por ideas e intereses divergentes, malos o sobre entendidos. El problema está en cómo se tramita, si se utiliza la agresividad, se evade o se utilizan instrumentos mediadores y conciliadores. Si se aborda con autoritarismo, con exclusión y castigo. Esto hace que se convierta en un problema y que provoque tensiones y rivalidades sin que se puedan resolver; problemas ocultos a las relaciones interpersonales, en el currículo escolar y por qué no en el clima institucional.

\section{RUTA PARA HACER FRENTE A LOS PROBLEMAS DE CONVIVENCIA ESCOLAR}

Entre las estrategias y procedimientos institucionales de la comunidad educativa dadas a partir del manual de convivencia y rol que cumplen las coordinaciones y las direcciones de grupo se describe en la siguiente una ruta institucional: 


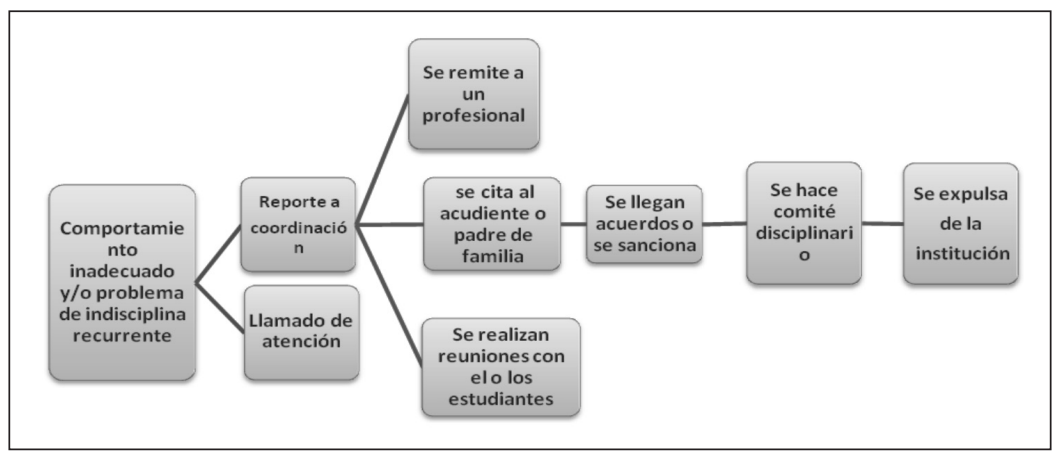

Figura 1. Ruta de atención institucional Fuente: Esta investigación

La vigencia de un modelo educativo centrado en la obediencia conlleva a que algunos estudiantes sean sumisos y otros rebeldes. Se valida el reporte, la sanción, el castigo y la expulsión como formas eficaces de hacer que se cumplan las normas. Esto nos indica que el manual de convivencia se utiliza más para sancionar que para regular la convivencia. Los líderes estudiantes no juegan un papel protagónico en las aulas escolares. Solo se les consulta o se les informa las determinaciones a seguir.

\section{CONCLUSIONES}

Indagar la convivencia escolar no es cuestión de una o varias visitas, encuentros o reuniones, es un proceso que exige el vínculo permanente entre los actores a fin de describir, comprender y transformar interacciones interpersonales e intergrupales. Más aún comprender las dinámicas de la normatividad escolar y los procesos de enseñanza - aprendizaje que contribuyen o no al desarrollo de comportamientos agresivos o excluyentes al interior de las aulas escolares. La convivencia al interior de esta institución educativa se concibe de una manera positiva que puede ser irrumpida por los problemas de comportamiento e indisciplina de los estudiantes además de la inadecuada tramitación del conflicto. Se resaltan las relaciones armoniosas como un proceso que se construye a partir de las emociones del otro, de su actitud y del lenguaje que se use.

Dentro de los problemas de convivencia más comunes y generalizados en el contexto escolar observado se identifican las prácticas y relaciones agresivas entre pares, la agresión verbal, la presencia de objetos que interfieren en el aula escolar (celulares, maquillaje, ruido), la indisciplina, el incumplimiento de las normas de disciplina, las riñas y alegatos entre algunos estudiantes además de la poca concentración de los estudiantes en las aulas escolares. Aspectos que deben ser abordados desde aprendizajes cooperativos para el desarrollo de habilidades y de competencias personales y grupales. 
Entre los factores influyentes de estos problemas se reconocen las relaciones intrafamiliares, los grupos de pares y las amistades el cual los estudiantes mantienen contacto en sus horas extraescolares. Se resalta cómo el escenario escolar es el reflejo de lo que sucede en la familia, la comunidad y por ende la sociedad en general. Desde la práctica docente se identifica que algunos docentes ejercen la autoridad de manera rígida a gritos, otros son pasivos y receptivos, se limitan a dictar sus clases, mientras que otros son amistosos, consejeros y críticos de las interacciones cotidianas y de los problemas que vivencia los estudiantes en sus aulas escolares. De allí que sea necesario abordar el rol del docente y su práctica pedagógica.

Los estudiantes presentan necesidades de afecto y apoyo psicológico para abordar sus crisis emocionales y familiares. Además, se evidencia la necesidad de potenciar su autonomía y compromiso de los estudiantes frente a sus responsabilidades y tareas asignadas. Es importante trabajar el desarrollo de habilidades sociales y comunicativas que permitan una mejor interacción con el otro.

En cuanto al manual de convivencia este se concibe como un instrumento útil y construido con cierta participación de la comunidad. Se socializa y se reflexiona con la comunidad estudiantil a partir del área de ética y las direcciones de grupo. Los representantes estudiantiles no evidencian un papel protagónico en sus grupos ni en la institución educativa. Son tenidos en cuenta para participar en reuniones instituciones pero no desarrollan un propio plan de trabajo.

Esta experiencia investigativa ha sido un proceso enriquecedor porque se han compartido experiencias y conocimientos, se identifican las potencialidades y aspectos del equipo de trabajo por mejorar y se pone a flote el desarrollo de habilidades de indagación, de registro, liderazgo y creatividad en el proceso de la investigativa formativa. Además, ha permitido un acercamiento a la realidad social que viven los docentes y estudiantes en el ámbito escolar.

\section{REFERENCIAS BIBLIOGRÁFICAS}

ÁlVARO. José Luís. GARIDO Alicia. OVEJERO Anastasio. Psicología social de la educación en Psicología Social Aplicada. J. R. Torregrosa. MCgrawllil. Interamericana de España, S.A. U. Madrid. 1999.

GARNICA, FONSECA, GONZALES, RODRÍGUEZ, SIERRA y VELASCO. Comprendiendo la agresividad escolar como punto de partida para formar ciudadanía. Instituto de investigación educativa y desarrollo pedagógico IDEP. Proyecto para generar procesos de aprendizaje ciudadano en estudiantes de básica y media vocacional. Bogotá. Abril del 2005.

LUCA DE TENA DE BETHENCOURT, María del Carmen ; RODRÍGUEZ R, Rosa Isabel. La acción tutorial para favorecer la convivencia: la disciplina. Universitat de les Illes Balears. Palma de Mallorca, Conselleria de Educación y Cultura, 2001. 
MARTÍN. Baró. Ignacio. Acción e ideología. UCA Editores. El salvador. 1997. Presidencia de la república. Oficina del Alto comisionado para la Paz. Cartilla promotores de convivencia. Bogotá, D. C., Colombia. Junio de 2004.

MUÑOZ QUEZADA, María Teresa, SAAVEDRA G., Eugenio y VILLALTA P., Marco. Percepciones y significados sobre la convivencia y violencia escolar de estudiantes de cuarto medio de un liceo municipal de Chile. Rev. Ped. [Online]. 2007, vol.28, no.82 [citado 20 Agosto 2009], p.197-224. Disponible en la World Wide Web: <http:/www.scielo.org. ve/scielo.php?script=sci_arttext\&pid=S0798-97922007000200003\&lng=es\&nrm=iso>. ISSN 0798-9792.

NORMAN J. de Jesús, M. ED. La violencia y el sistema escolar. Santa Isabel Puerto Rico. Mayo de 2005. Extraído el 5 de marzo de 2008 de http://www.cyberpediatria.com/

SIERRA, Gonzalo. Escuela, cultura y comunidad. Editorial El Búho LTDA. 2004. Pág. 53 y 105.

TORRES CARRILLO, Alfonso. Aprender a investigar en comunidad II enfoques cualitativos y participativos en la comunicación social. Bogotá, Facultad de Ciencias Sociales y Humanas de Unisur, 1995. 